\title{
Caracterización de la respuesta ovárica a la superovulación en bovino Criollo Coreño utilizando dosis reducidas de FSH
}

\section{Characterization of the ovarian response to superovulation in Creole Coreño cattle using reduced doses of FSH}

\author{
Fernando Villaseñor González ${ }^{\mathrm{a}}$, José Fernando de la Torre Sánchez ${ }^{\mathrm{b}}$, Guillermo Martínez Velázquez ${ }^{*}$,

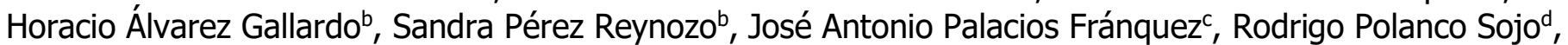 \\ Moisés Montaño Bermúdez ${ }^{e}$
}

RESUMEN

Para evaluar la respuesta ovárica a la superovulación con dosis reducidas de la hormona folículo estimulante (FSH) en bovinos Criollo Coreño, se realizaron dos experimentos en el Sitio Experimental El Verdineño del INIFAP ubicado en Nayarit, México. Se utilizaron 12 vacas de $12.4 \pm 2.9$ años (Exp 1) y seis vaquillas de $3.0 \pm 0.3$ años (Exp 2). Se aplicaron tres tratamientos a cada hembra: $280 \mathrm{mg}$ (T1), $200 \mathrm{mg}$ (T2) y $140 \mathrm{mg}$ (T3) de FSH. Las variables evaluadas fueron número de embriones transferibles (ET), número de corpúsculos recuperados $(\mathrm{CR})=($ embriones + óvulos no fertilizados), número de embriones no transferibles (ENT), número de cuerpos lúteos (CL), número de óvulos no fertilizados (ONF), volumen ovárico (VO), concentración sérica de progesterona (P4), porcentaje de fertilización $(\% F)=((E T+E N T) * 100) / C R)$ y porcentaje de recuperación $(\% R)=C L * 100 / C R$. Se utilizó un diseño experimental crossover simple balanceado. La información se analizó con el procedimiento GLM de SAS. El modelo estadístico incluyó los efectos de tratamiento (T1, T2 y T3), vaquilla/vaca (6 y 12) y período (3 períodos). En el Exp 1 se detectaron diferencias $(P<0.05)$ entre tratamientos para CR y ONF favorables a T1. No se encontraron diferencias $(P>0.05)$ entre tratamientos para ET, ENT, CL, VO, P4, \%F o \%R. En el Exp 2 no se detectaron diferencias $(P>0.05)$ entre tratamientos para ninguna de las variables. Es factible utilizar dosis reducidas de FSH para la superovulación en vaquillas Criollo Coreño sin afectar la respuesta a la superovulación o la producción de embriones.

PALABRAS CLAVE: Bovinos, Superovulación, Embriones transferibles, Criollo Coreño.

\section{ABSTRACT}

To evaluate the ovarian response to superovulation using reduced doses of FSH in Criollo Coreño cattle two experiments were conducted at the Experimental Station "EI Verdineño" (INIFAP) located in Nayarit, México. Twelve (12) cows of 12.4 \pm 2.9 yr old (Exp 1) and six heifers of $3.0 \pm 0.3 \mathrm{yr}$ old (Exp 2) were used. Three treatments were assigned to each female consisting of $280 \mathrm{mg}$ (T1), $200 \mathrm{mg}$ (T2) and $140 \mathrm{mg}$ (T3) of FSH, so that all females received all treatments. The response variables were transferable embryos (ET), corpuscles retrieved (CR) $=($ embryos+non fertilized ova), degenerated embryos (ENT), number of corpora lutea (CL), non-fertilized ova (ONF), ovarian volume (VO), serum progesterone $(\mathrm{P4})$, fertilization rate $(\% \mathrm{~F})=((\mathrm{ET}+\mathrm{ENT}) * 100) / \mathrm{CR})$ and percent recovery $(\% \mathrm{R})=\mathrm{CL} * 100 / \mathrm{CR}$. A cross-over balanced experimental design was used. Analyses were carried out with the GLM procedure of SAS. The statistical model included the fixed effects of treatment (T1, T2 and T3), animal (twelve or six) and period (three periods). In Exp 1 differences among treatments $(P<0.05)$ favorable to T1 were detected for CR and ONF. No differences were found $(P>0.05)$ among treatments for ET, ENT, CL, VO, P4, \%F or \%R. In Exp 2 no differences were detected $(P>0.05)$ among treatments for any of the variables. It is feasible to use reduced doses of FSH for the superovulation of Criollo Coreño heifers without affecting the response to superovulation or to embryo production.

KEY WORDS: Cattle, Superovulation, Transferable embryos, Criollo Coreño.

\footnotetext{
Recibido el 4 de abril de 2016. Aceptado el 10 de noviembre de 2016.

a Campo Experimental Centro Altos de Jalisco. Instituto Nacional de Investigaciones Forestales, Agrícolas y Pecuarias (INIFAP). México.

b Centro Nacional de Recursos Genéticos, INIFAP. Tepatitlán, Jalisco, México.

c Sitio Experimental "El Verdineño", INIFAP. México.

d Práctica privada. México.

e Centro Nacional de Investigación Disciplinaria en Fisiología y Mejoramiento Animal. INIFAP. México.

*Autor de correspondencia: martinez.guillermo@inifap.gob.mx.
} 


\section{INTRODUCCIÓN}

Los bovinos criollos son poblaciones resistentes a enfermedades parasitarias, están adaptados a climas extremos y poseen gran rusticidad y resiliencia ${ }^{(1)}$. Estas poblaciones pueden contribuir a la producción eficiente de becerros para la engorda al utilizarse en programas de cruzamientos, especialmente como líneas maternas ${ }^{(2,3)}$.

En México se han identificado núcleos de bovinos criollos que se encuentran en zonas aisladas, agrestes, poco comunicadas y de difícil acceso; son zonas donde por lo general habitan pueblos indígenas ${ }^{(1,4)}$. Dichos núcleos son reducidos en número de cabezas y se encuentran disminuyendo día con día ${ }^{(5,6)}$. De acuerdo a la FAO, es importante la conservación de estos recursos zoogenéticos como parte de la preservación de la diversidad genética de cada país ${ }^{(7)}$.

Para la conservación de los recursos zoogenéticos la conservación in vitro de gametos y embriones juega un papel relevante. La superovulación y la transferencia de embriones son biotecnologías reproductivas que utilizan gonadotropinas exógenas y permiten mejorar la tasa reproductiva de las vacas donadoras ${ }^{(8)}$. Un factor fundamental para el éxito de la transferencia de embriones es el desarrollo de protocolos de superovulación adecuados a la raza, talla y función zootécnica de la donadora. La necesidad de utilizar variantes en los protocolos de superovulación obedece a las diferencias fisiológicas que existen entre las razas de las donadoras ${ }^{(9)}$. Estudios realizados en el estado de Chihuahua, México, mostraron que la mayoría de las hembras criollas evaluadas presentaron dos ondas de desarrollo folicular por ciclo, además de que la secreción de gonadotropinas y la funcionalidad ovárica en estas hembras fueron diferentes a las de otras razas, tanto Bos taurus como Bos indicus ${ }^{(10,11)}$.

En general, las razas de bovinos criollos son animales de talla mediana o pequeña, de bajos requerimientos nutricionales y de función zootécnica no demandante. Estas características parecen favorecer la respuesta superovulatoria con dosis menores de la hormona folículo estimulante (FSH), en comparación a las dosis que se utilizan comercialmente en ganado bovino productor de carne ${ }^{(12-15)}$.

En México, en la región de la Sierra Madre Occidental que comparten los estados de Durango, Jalisco, Nayarit y Zacatecas existen hatos de animales localmente adaptados denominados Criollo Coreño( ${ }^{(4,16)}$. Estos animales son de talla mediana o pequeña y se cuenta con información sobre su comportamiento productivo y reproductivo ${ }^{(3,10,11)}$; sin embargo, falta documentar su respuesta a la superovulación y producción de embriones. Cabe señalar que generar información al respecto permitirá desarrollar protocolos de superovulación para este tipo de ganado, contribuyendo así a caracterizar su comportamiento reproductivo.

Por lo anterior y como una contribución a la caracterización del comportamiento reproductivo del ganado Criollo Coreño, el presente estudio se planteó con el objetivo de evaluar la respuesta ovárica a la superovulación de vacas y vaquillas utilizando tres dosis de hormona FSH. Lo anterior bajo la hipótesis de que dosis reducidas de FSH de 200 y 140 mg aplicadas en hembras Criollo Coreño no son diferentes de la dosis de $280 \mathrm{mg}$ para la estimulación de ovulaciones múltiples y la producción de embriones.

\section{MATERIAL Y MÉTODOS}

El estudio se realizó en el Sitio Experimental El Verdineño del Instituto Nacional de Investigaciones Forestales Agrícolas y Pecuarias (INIFAP-SAGARPA), que se localiza en el municipio de Santiago Ixcuintla Nayarit, México. El clima es tropical cálido subhúmedo $A w 2^{(17)}$. Se encuentra a una altitud de $50 \mathrm{msnm}$ con temperatura media anual de $25^{\circ} \mathrm{C}$ y precipitación pluvial promedio de $1,200 \mathrm{~mm}^{(18)}$.

\section{Manejo de los animales}

Se llevaron a cabo dos experimentos con vacas y vaquillas Criollo Coreño. El Exp 1 se realizó entre junio y octubre del año 2013 utilizándose 12 vacas con edad promedio de $12.4 \pm 2.9$ años (desde 9 hasta 17 años). El Exp 2 se realizó entre abril y julio del año 2014 utilizándose seis vaquillas con edad promedio de $3.0 \pm 0.3$ años (desde 2 hasta 3 años). 
Previo al inicio de cada experimento a las hembras se les realizó un examen físico general que incluyó la evaluación de la morfología e integridad del tracto reproductor. Se utilizó un ultrasonido portátil con un transductor rectal de 7.5 $\mathrm{MHz}$ para confirmar la ausencia de preñez, evaluar la condición ovárica y verificar la ausencia de quistes, adherencias y otras patologías; adicionalmente, se confirmó la ausencia de fibrosis y malformaciones pasando a través del cérvix un estilete. La condición corporal se evaluó utilizando una escala del 1 al 9 en donde 1 correspondió a un animal extremadamente emaciado y 9 a un animal muy obeso ${ }^{(19)}$. Las vacas del primer experimento tuvieron condición corporal inicial y final de $5.8 \pm 0.6$ y $6.4 \pm 0.7$, mientras que las vaquillas del segundo experimento tuvieron condición corporal inicial y final de $6.0 \pm 0.1$ y $6.4 \pm$ 0.5 .

En ambos experimentos se aplicó en tres ocasiones el mismo protocolo de superovulación (SO). Previo a la primera SO las hembras se sometieron a la sincronización de la oleada de desarrollo folicular para hacer coincidir la aparición de una nueva oleada con el inicio del protocolo de SO. Este consistió en la aplicación de 2 y $5 \mathrm{mg}$ de benzoato de estradiol para vaquillas y vacas, respectivamente (Syntex $囚)$, más $100 \mathrm{mg}$ de progesterona (P4) (Pfizer $囚)$, y la inserción de un dispositivo intravaginal conteniendo $1 \mathrm{~g}$ de P4 (Syntex®). Al quinto día, se inició la SO con la aplicación de FSH (Folltropin- $\mathrm{V}^{\circledR}$ ) de manera fraccionada y decreciente durante cuatro días en dos aplicaciones diarias con intervalos de $12 \mathrm{~h}$, retirando el implante de P4 al tercer día de dicho tratamiento. Al quinto día de iniciada la SO se detectaron estros y se inseminó, a 12, 24 y 36 h pos estro con semen de uno de cuatro toros Criollo Coreño, a cada vaca o vaquilla.

La colecta de los embriones se realizó siete días después de detectado el celo. Para los tratamientos subsecuentes se utilizó un esquema de reciclado rápido de donadoras para reducir el intervalo entre SO a 33-38 días, que consistió en lo siguiente: al finalizar la recuperación de embriones, se aplicaron $25 \mathrm{mg}$ de PGF2a y un dispositivo intravaginal de progesterona; 10 días después, se retiró el dispositivo y se aplicó PGF2a, tres días después; se vigiló a las donadoras para detectar el comportamiento de estro y 12 días después de éste se inició un nuevo protocolo de $\mathrm{SO}^{(20)}$.

\section{Alimentación}

En ambos experimentos las hembras fueron confinadas y alimentadas con una dieta que contenía $15.5 \%$ de proteína cruda y $2.8 \mathrm{Mcal} / \mathrm{Kg}$ de EM. Vacas y vaquillas fueron alimentadas con un concentrado que contenía alfalfa, sorgo, pasta de canola, salvado de trigo, melaza, urea, sal granulada, minerales comerciales, levaduras comerciales y grasas de sobrepaso. Se ofrecieron de 3 a $4 \mathrm{~kg}$ de concentrado por día dependiendo de la condición corporal y si tenía o no cría al pie. Las fuentes de forraje que se utilizaron fueron pajas amoniatizadas de frijol (Phaseolus vulgaris) y de pasto pangola (Digitaria decumbens) a libre acceso. Esta dieta se ofreció dos meses antes de iniciar con la sincronización y superovulación de las hembras y durante toda la fase experimental.

\section{Mediciones antes de la colección de embriones}

Previo a la colección de embriones se escanearon los ovarios mediante ultrasonido para contar los cuerpos lúteos presentes en cada ovario. El volumen ovárico se estimó midiendo cada ovario a lo largo, ancho y profundo. De cada hembra se tomó una muestra de sangre para obtener suero y determinar por radioinmunoanálisis la concentración sérica de $\mathrm{P} 4$ circulante el séptimo día después del estro. Para el análisis de P4 se utilizó un kit comercial (DPC, Los Angeles CA) con una sensibilidad de 0.03 $\mathrm{ng} / \mathrm{ml}$ y coeficiente de variación intra-ensayo e interensayo de 4.5 y $8.8 \%$, respectivamente.

\section{Colección de embriones}

Las donadoras se colocaron en una trampa inmovilizadora en donde se lavó y desinfectó el área perivulvar y coccígea dorsal, aplicándoseles entre 5 y $8 \mathrm{ml}$ de lidocaína al $2 \%$ por vía epidural. Se aplicó xilazina al $2 \%$ como tranquilizante cuando fue necesario. Se insertó una sonda Foley de dos vías por vía vaginal, fijándola en el cuerpo del útero, para realizar las infusiones-colecciones uterinas. Se utilizaron $1.5 \mathrm{~L}$ de solución amortiguadora fosfatada, 
suplementada con glucosa, piruvato, antibióticos y $0.3 \%$ de alcohol polivinílico (PBSm) $)^{(21)}$.

\section{Búsqueda, evaluación y criopreservación de embriones}

Se vació el contenido del filtro en una caja Petri cuadrada de $100 \times 100 \mathrm{~mm}$, con fondo cuadriculado para la búsqueda de embriones en el microscopio con un aumento de 20 a 30X. Se preparó una caja Petri de $35 \mathrm{~mm}$ con solución de mantenimiento (PBSm con albúmina sérica bovina) para colocar los embriones que se iban encontrando. La evaluación morfológica se realizó según los criterios del manual de la sociedad internacional de transferencia de embriones(22). Los embriones se catalogaron de acuerdo a su estadio de desarrollo en una escala del 1 (estadio de una célula) al 9 (estadio de blastocito eclosionado) y según su calidad como 1 (excelente), 2 (bueno), 3 (regular) y 4 (degenerado). Para la criopreservación los embriones en estadio de mórula y blastocito con calidades 1 y 2 se pusieron en medio de congelación con etilén glicol al $10 \%, 0.3 \mathrm{M}$ de sacarosa, y se empajillaron. Posteriormente se pusieron en una congeladora automática, la cual descendió la temperatura de manera gradual y automática desde temperatura ambiente hasta llegar a los $-6^{\circ} \mathrm{C}$, a una tasa de $3^{\circ} \mathrm{C} / \mathrm{min}$; a esta temperatura se sembró la cristalización para lograr un congelamiento uniforme en la parte donde se localizaba el embrión; la temperatura siguió entonces descendiendo a una tasa de $0.5^{\circ} \mathrm{C} / \mathrm{min}$, hasta llegar a los $-35{ }^{\circ} \mathrm{C}$, y una vez a esta temperatura se pasaron directo al tanque de nitrógeno líquido $\mathrm{a}-196^{\circ} \mathrm{C}$.

\section{Variables, tratamientos y periodos}

En ambos experimentos las variables de respuesta evaluadas fueron número de embriones transferibles (ET), número de corpúsculos recuperados $(C R)=$ (embriones + óvulos no fertilizados), número de embriones no transferibles (ENT), número de cuerpos lúteos $(C L)$, número de óvulos no fertilizados (ONF), volumen ovárico (VO), concentración sérica de $\mathrm{P} 4$, porcentaje de fertilización $(\% \mathrm{~F})=((\mathrm{ET}+\mathrm{ENT}) * 100) / \mathrm{CR}$ y porcentaje de recuperación $(\% \mathrm{R})=\mathrm{CL} * 100 / \mathrm{CR}$. Los tratamientos consistieron en aplicar tres dosis de una preparación estandarizada de FSH-LH (Folltropin-V囚) con dosis totales de $280 \mathrm{mg}$ de FSH (T1), 200 mg de FSH (T2) y 140 mg de FSH (T3). Cada unidad experimental (vaca 0 vaquilla donadora) recibió los tres tratamientos en tres diferentes periodos.

\section{Análisis estadístico}

Los datos se analizaron con el procedimiento de modelos lineales generalizados (PROC GLM) del paquete estadístico SAS ${ }^{(23)}$. Lo anterior considerando que existen estudios que mencionan diferencias mínimas en la estimación de parámetros generados al analizar una misma característica como continua o discreta(24,25,26). El diseño experimental utilizado fue un crossover simple balanceado(27) con el siguiente modelo estadístico:

$$
\mathrm{Y}_{\mathrm{ijkl}}=\mu+a_{i}+\beta_{j}+\mathrm{T}_{\mathrm{k}}+\varepsilon_{\mathrm{ijkl}}
$$

En donde $Y_{\mathrm{ijk}}$ es la k-ésima observación de la variable de respuesta; $\mu=$ media general; $a_{i=}$ efecto de la i-ésima hembra ( $i=1,2 \ldots 6$ ó 12$) ; \beta_{j=}$ efecto del j-ésimo periodo $(j=1,2,3) ; T_{k=}$ efecto del k-ésimo tratamiento $(\mathrm{k}=1,2,3) ; \quad \varepsilon_{\mathrm{ijk}}=$ error experimental distribuido NI $\left(0, \sigma^{2} \mathbf{e}\right)$.

Se estimaron coeficientes de correlación de Pearson entre la variable P4 y las variables $C L$ y VO utilizando el procedimiento PROC CORR del paquete estadístico SAS ${ }^{(23)}$.

\section{RESULTADOS Y DISCUSIÓN}

\section{Experimento 1}

Promedios no ajustados. El $44 \%$ de las vacas produjeron al menos un embrión transferible, $8 \%$ de las mismas no presentó respuesta superovulatoria a las gonadotropinas exógenas. El $33 \%$ de las vacas produjeron embriones transferibles durante dos periodos distintos, el $50 \%$ sólo produjeron embriones transferibles en un periodo. El $8 \%$ de las vacas no produjeron ningún embrión transferible durante los tres periodos de la fase experimental.

En el Cuadro 1 se presentan las medias de cuadrados mínimos y los errores estándar para las diferentes variables evaluadas. Se encontraron 
Cuadro 1. Medias de cuadrados mínimos y errores estándar de vacas Criollo Coreño para número de embriones transferibles (ET), número de corpúsculos recuperados (CR), número de embriones no transferibles (ENT), número de cuerpos lúteos $(\mathrm{CL})$, número de óvulos no fertilizados (ONF), volumen ovárico $(\mathrm{VO})$, concentración sérica de progesterona $(\mathrm{P} 4)$, porcentaje de fertilización $(\% \mathrm{~F})$ y porcentaje de recuperación $(\% \mathrm{R})$, considerando tres tratamientos ${ }^{\mathrm{TT}}$ y tres períodos $^{\mathrm{PP}}$

\begin{tabular}{ccccccc}
\hline Variable & Tratamiento 1 & Tratamiento 2 & Tratamiento 3 & Período 1 & Período 2 & Período 3 \\
\hline ET & $1.2 \pm 0.7^{\mathrm{a}}$ & $2.9 \pm 0.7^{\mathrm{a}}$ & $1.1 \pm 0.7^{\mathrm{a}}$ & $2.4 \pm 0.7^{\mathrm{a}}$ & $1.6 \pm 0.7^{\mathrm{a}}$ & $1.2 \pm 0.7^{\mathrm{a}}$ \\
$\mathrm{CR}$ & $10.3 \pm 1.7^{\mathrm{a}}$ & $7.2 \pm 1.7^{\mathrm{a}}$ & $4.0 \pm 1.7^{\mathrm{b}}$ & $6.3 \pm 1.7^{\mathrm{a}}$ & $9.6 \pm 1.7^{\mathrm{a}}$ & $5.6^{\mathrm{a}} 1.7^{\mathrm{a}}$ \\
$\mathrm{ENT}$ & $2.0 \pm 0.5^{\mathrm{a}}$ & $1.8 \pm 0.5^{\mathrm{a}}$ & $0.8 \pm 0.5^{\mathrm{a}}$ & $1.2 \pm 0.5^{\mathrm{a}}$ & $2.0 \pm 0.5^{\mathrm{a}}$ & $1.6^{\mathrm{a}} 0.5^{\mathrm{a}}$ \\
$\mathrm{CL}$ & $10.4 \pm 0.7^{\mathrm{a}}$ & $9.1 \pm 0.7^{\mathrm{a}}$ & $8.8 \pm 0.7^{\mathrm{a}}$ & $7.8 \pm 0.7^{\mathrm{a}}$ & $9.9 \pm 0.7^{\mathrm{ab}}$ & $10.5^{\mathrm{a}} 0.7^{\mathrm{b}}$ \\
$\mathrm{ONF}$ & $7.3 \pm 1.6^{\mathrm{a}}$ & $2.4 \pm 1.6^{\mathrm{ab}}$ & $2.1 \pm 1.6^{\mathrm{b}}$ & $2.9 \pm 1.6^{\mathrm{a}}$ & $6.0 \pm 1.6^{\mathrm{a}}$ & $2.8^{\mathrm{a}} 1.6^{\mathrm{a}}$ \\
$\mathrm{VO}, \mathrm{cm}$ & $14.8 \pm 1.5^{\mathrm{a}}$ & $11.8^{3} \pm 1.5^{\mathrm{a}}$ & $11.0 \pm 1.5^{\mathrm{a}}$ & $11.5 \pm 1.5^{\mathrm{a}}$ & $11.1 \pm 1.5^{\mathrm{a}}$ & $14.1 \pm 1.5^{\mathrm{a}}$ \\
$\mathrm{P} 4, \mathrm{ng} / \mathrm{ml}$ & $11.7 \pm 1.8^{\mathrm{a}}$ & $8.7^{\mathrm{a}} 1.8^{\mathrm{a}}$ & $7.7 \pm 1.8^{\mathrm{a}}$ & $5.2 \pm 1.8^{\mathrm{a}}$ & $11.0 \pm 1.8^{\mathrm{ab}}$ & $11.9^{\mathrm{a}} 1.8^{\mathrm{b}}$ \\
$\% \mathrm{~F}$ & $44.6 \pm 10.4^{\mathrm{a}}$ & $52.5 \pm 11.9^{\mathrm{a}}$ & $36.9 \pm 11.1^{\mathrm{a}}$ & $32.1 \pm 10.4^{\mathrm{a}}$ & $53.1 \pm 11.1^{\mathrm{a}}$ & $48.8^{\mathrm{a}} \pm 11.9^{\mathrm{a}}$ \\
$\% \mathrm{R}$ & $79.4 \pm 11.7^{\mathrm{a}}$ & $57.1 \pm 11.7^{\mathrm{a}}$ & $43.6 \pm 11.7^{\mathrm{a}}$ & $56.8 \pm 11.7^{\mathrm{a}}$ & $80.7 \pm 11.7^{\mathrm{a}}$ & $42.6 \pm 11.7^{\mathrm{a}}$ \\
\hline
\end{tabular}

$\mathrm{CR}=($ embriones $+\mathrm{ONF}) ; \% \mathrm{~F}=\left((\mathrm{ET}+\mathrm{ENT}){ }^{*} 100\right) / \mathrm{CR} ; \% \mathrm{R}=\mathrm{CL}{ }^{\star} 100 / \mathrm{CR}$.

TT Tratamiento 1= $280 \mathrm{mg}$ de FSH; Tratamiento 2= $200 \mathrm{mg}$ de FSH; Tratamiento 3= $140 \mathrm{mg}$ de FSH.

PP Entre 33 y 38 días la duración de cada período.

abc Diferentes literales en el mismo renglón indican diferencias $(P<0.05)$ entre tratamientos o entre períodos.

diferencias $(P<0.05)$ entre tratamientos para CR y ONF favorables a T1; sin embargo, no se detectaron diferencias importantes $(P>0.05)$ entre tratamientos para ET, ENT, CL, VO, P4, \%F o \%R. Cabe señalar que los promedios para ENT, $\mathrm{CL}, \mathrm{ONF}$, VO y \%R sugieren a T1 como el tratamiento más determinante para favorecer la superovulación en vacas. El periodo influyó significativamente $(P<0.05)$ sobre las variables $C L$ y $P 4$; en contraste, no se detectaron diferencias estadísticas entre periodos para las variables $\mathrm{ET}, \mathrm{CR}, \mathrm{ENT}, \mathrm{ONF}, \mathrm{VO}, \% \mathrm{~F}$ o \%R.

En las vacas del Exp 1 se observó un comportamiento descendente para $\mathrm{CR}$, a medida que disminuyó la dosis de $\mathrm{FSH}$, detectándose la mayor respuesta en T1 $(10.3 \pm 1.7 \mathrm{CR})$ que fue diferente $(P<0.05)$ de T3 (4.1 $\pm 1.7 \mathrm{CR})$. En contraste, se encontró que a T1 (280 mg de FSH) correspondió la mayor cantidad de ONF, lo que fue diferente $(P<0.05)$ de T3 $(140 \mathrm{mg}$ de FSH). Lo anterior mostró que la dosis reducida (T3) promovió una menor respuesta superovulatoria en las vacas comparada con la dosis estándar T1; sin embargo, lo anterior no se reflejó en una mayor producción de embriones para T1 por la gran cantidad de ONF encontrados en este tratamiento. La cantidad de corpúsculos recuperados en este experimento fue similar a los promedios reportados en otros estudios realizados con ganado $B$. taurus y $B$. indicus ${ }^{(9,28)}$, en los que se utilizaron las dosis estándar recomendadas para bovinos carne y que oscilan entre 260 y $280 \mathrm{mg}$ de $\mathrm{FSH}^{(29)}$.

La alta proporción de ONF con respecto a los corpúsculos totales recuperados en T1 puede atribuirse a la edad (12.4 \pm 2.93 años) de las donadoras que se utilizaron en el Exp 1, puesto que hay información publicada que establece que la producción de embriones decrece después de los 8 años de edad ${ }^{(30)}$. Lo anterior no se atribuye a una disminución en la tasa ovulatoria, sino a una disminución en la tasa de fertilización y calidad de los embriones ${ }^{(31)}$. En el presente estudio las donadoras entre 9 y 12 años de edad tuvieron una tasa de fertilización del $49 \%$, mientras que aquéllas que tenían de 14 a 17 años solo tuvieron el $27 \%$ de fertilización. En relación a las causas de la baja tasa de fertilización en hembras superovuladas de edad avanzada, existen estudios que indican que en este tipo de animales la FSH y el estradiol aumentan sus niveles séricos provocando fallas en la maduración y crecimiento de los folículos, comprometiendo la ovulación, la fertilización y el adecuado desarrollo embrionario(32). Por otro lado, también se ha encontrado que el número de ovocitos se limita en hembras con 9 o más años de edad ${ }^{(20)}$, debido a que el desarrollo y maduración de los ovocitos se afecta desfavorablemente conforme se incrementa la edad 
de las vacas, disminuyendo su capacidad de ser fertilizados $^{(33)}$. Así, estudios realizados con hembras de diferentes razas y edades han establecido que hembras jóvenes tienen mayores porcentajes de blastocitos, comparadas con hembras de mayor edad con 32.5 y $22.8 \%$ de blastocitos para vaquillas y vacas, respectivamente ${ }^{(34)}$ y $45.9,30.2$ y $13.5 \%$ de blastocitos para vaquillas de 12 meses de edad, vacas de 7 y 8 años de edad y vacas mayores de 15 años de edad, respectivamente ${ }^{(30)}$. Por otro lado, considerando que en el presente estudio a cada vaca o vaquilla se le dieron tres servicios de inseminación artificial a las 12,24 y 36 h después de detectado el estro con semen de calidad certificada, se descartó como causa de la baja fertilización deficiencias en el proceso de inseminación.

\section{Experimento 2}

Promedios no ajustados. El $52 \%$ de las vaquillas produjeron al menos un embrión transferible. El $26 \%$ de vaquillas no presentaron respuesta superovulatoria a las gonadotropinas exógenas y en el $42 \%$ no se recuperó estructura alguna. El $33 \%$ de las vaquillas produjeron embriones transferibles durante dos periodos distintos y $33 \%$ de vaquillas sólo produjeron embriones transferibles en un periodo. El $16 \%$ de las vaquillas no produjeron ningún embrión transferible en ninguno de los tres periodos.
En el Cuadro 2 se presentan las medias de cuadrados mínimos y los errores estándar para las variables evaluadas en vaquillas. No se detectaron diferencias estadísticas entre tratamientos o entre periodos ( $P>0.05$ ) para ninguna de las variables analizadas. La variable ONF no fue estimada debido a los escasos datos disponibles. Cabe señalar que la no diferencia entre tratamientos para ninguna de las variables evaluadas muestra la factibilidad de utilizar dosis reducidas de FSH en vaquillas Criollo Coreño sin afectar desfavorablemente su respuesta a la superovulación.

\section{Contrastes entre ambos experimentos}

Para el total de hembras se encontró una correlación positiva entre $\mathrm{CL}$ y P4, lo que se reflejó en que las hembras con mayor número de cuerpos lúteos fueron las que presentaron los niveles más altos $(P<0.05)$ de progesterona. También se detectó una correlación positiva entre VO y P4 lo que significó que las hembras con mayor volumen ovárico presentaron también la mayor producción de progesterona $(P<0.05)$.

Considerando los resultados de los tres tratamientos en ambos experimentos, se observó que las vacas respondieron mejor a la superovulación al producir más cuerpos lúteos (10.4

Cuadro 2. Medias de cuadrados mínimos y errores estándar de vaquillas Criollo Coreño para número de embriones transferibles (ET), número de corpúsculos recuperados (CR), número de embriones no transferibles (ENT), número de cuerpos lúteos (CL), volumen ovárico (VO), concentración sérica de progesterona (P4), porcentaje de fertilización (\%F) y porcentaje de recuperación $(\% \mathrm{R})$, considerando tres tratamientos ${ }^{\mathrm{TT}}$ y tres períodos ${ }^{\mathrm{PP}}$

\begin{tabular}{cccc|ccc}
\hline Variable & Tratamiento 1 & Tratamiento 2 & Tratamiento 3 & Período 1 & Período 2 & Período 3 \\
\hline ET & $2.3 \pm 0.9^{\mathrm{a}}$ & $1.8 \pm 0.9^{\mathrm{a}}$ & $1.7 \pm 0.9^{\mathrm{a}}$ & $1.2 \pm 0.9^{\mathrm{a}}$ & $2.7 \pm 0.9^{\mathrm{a}}$ & $2.0 \pm 0.9^{\mathrm{a}}$ \\
$\mathrm{CR}$ & $3.8 \pm 1.1^{\mathrm{a}}$ & $2.3 \pm 1.1^{\mathrm{a}}$ & $2.0 \pm 1.1^{\mathrm{a}}$ & $2.5 \pm 1.1^{\mathrm{a}}$ & $3.0 \pm 1.1^{\mathrm{a}}$ & $2.7^{\mathrm{a}} 1.1^{\mathrm{a}}$ \\
$\mathrm{ENT}$ & $1.3 \pm 0.6^{\mathrm{a}}$ & $0.3 \pm 0.6^{\mathrm{a}}$ & $0.2 \pm 0.6^{\mathrm{a}}$ & $1.2 \pm 0.6^{\mathrm{a}}$ & $0.2 \pm 0.6^{\mathrm{a}}$ & $0.5 \pm 0.6^{\mathrm{a}}$ \\
$\mathrm{CL}$ & $5.8 \pm 1.1^{\mathrm{a}}$ & $5.5 \pm 1.1^{\mathrm{a}}$ & $4.5 \pm 1.1^{\mathrm{a}}$ & $3.8 \pm 1.1^{\mathrm{a}}$ & $5.8 \pm 1.1^{\mathrm{a}}$ & $6.2 \pm 1.1^{\mathrm{a}}$ \\
$\mathrm{VO}, \mathrm{cm}^{3}$ & $2.9 \pm 0.5^{\mathrm{a}}$ & $3.6 \pm 0.5^{\mathrm{a}}$ & $1.9 \pm 0.5^{\mathrm{a}}$ & ne & $3.4^{\mathrm{a}} 0.3^{\mathrm{a}}$ & $2.2^{\mathrm{a}} 0.3^{\mathrm{a}}$ \\
$\mathrm{P} 4 \mathrm{ng} / \mathrm{ml}$ & $9.1 \pm 2.3^{\mathrm{a}}$ & $6.7 \pm 2.3^{\mathrm{a}}$ & $5.0 \pm 2.3^{\mathrm{a}}$ & $7.5 \pm 2.3^{\mathrm{a}}$ & $8.1 \pm 2.3^{\mathrm{a}}$ & $5.3^{\mathrm{a}} 2.3^{\mathrm{a}}$ \\
$\% \mathrm{~F}$ & $96.5 \pm 3.8^{\mathrm{a}}$ & $95.7 \pm 3.5^{\mathrm{a}}$ & $97.3 \pm 3.9^{\mathrm{a}}$ & $94.0 \pm 4.9^{\mathrm{a}}$ & $94.0 \pm 3.8^{\mathrm{a}}$ & $94.8^{\mathrm{a}} 3.3^{\mathrm{a}}$ \\
$\% \mathrm{R}$ & $50.2 \pm 14.4^{\mathrm{a}}$ & $32.1 \pm 14.4^{\mathrm{a}}$ & $40.3 \pm 14.4^{\mathrm{a}}$ & $34.7 \pm 14.4^{\mathrm{a}}$ & $37.6 \pm 14.4^{\mathrm{a}}$ & $50.3 \pm 14.4^{\mathrm{a}}$ \\
\hline
\end{tabular}

ne= no estimable.

$\mathrm{CR}=($ embriones + óvulos no fertilizados $) ; \% \mathrm{~F}=((\mathrm{ET}+\mathrm{ENT}) * 100) / \mathrm{CR} ; \% \mathrm{R}=\mathrm{CL} * 100 / \mathrm{CR}$.

тT Tratamiento 1= $280 \mathrm{mg}$ de FSH; Tratamiento 2= $200 \mathrm{mg}$ de FSH; Tratamiento 3= $140 \mathrm{mg}$ de FSH.

PP Entre 33 y 38 días la duración de cada período.

abc Diferentes literales en el mismo renalón indican diferencias $(P<0.05)$ entre tratamientos 0 entre períodos. 
$\pm 0.7,9.1 \pm 0.7$ y $8.8 \pm 0.7$ para $\mathrm{T} 1, \mathrm{~T} 2$ y $\mathrm{T} 3$, respectivamente) que las vaquillas $(5.8 \pm 1.1,5.5 \pm$ 1.1 y $4.5 \pm 1.1$ para $T 1, \mathrm{~T} 2$ y $\mathrm{T} 3$, respectivamente), en contraste, las vacas tuvieron menor porcentaje de fertilización $(44.6 \pm 10.4,52.5 \pm 11.9$ y $36.9 \pm$ $11.1 \%$ para $\mathrm{T} 1, \mathrm{~T} 2$ y $\mathrm{T} 3$, respectivamente), que las vaquillas $(96.5 \pm 3.8,95.7 \pm 3.5$ y $97.3 \pm 3.9 \%$ para $T 1, T 2$ y $T 3$, respectivamente).

De igual manera y considerando los resultados de los tres tratamientos en ambos experimentos, se observó que las vacas mostraron promedios mayores que las vaquillas para VO, CL y P4. Coincidiendo con estos resultados, en otros estudios también se ha establecido una disminución en el número de ovulaciones, cuerpos lúteos y niveles de progesterona sérica en hembras jóvenes y de talla pequeña; debido a la sobre estimulación ovárica causada por los protocolos de superovulación, postulándose que hembras jóvenes superovuladas presentan fallas endocrinas debidas a las grandes cantidades de estradiol circulante, lo que provoca una baja respuesta a la superovulación ${ }^{(35)}$.

De acuerdo a los resultados para CR en los tres tratamientos en ambos experimentos, se observó que la respuesta ovulatoria fue menor en vaquillas $(3.8 \pm 1.1,2.3 \pm 1.1$ y $2.0 \pm 1.1 \mathrm{CR}$ para $\mathrm{T} 1, \mathrm{~T} 2$ y $\mathrm{T} 3$, respectivamente) comparada con la respuesta en las vacas $(10.3 \pm 1.7,7.2 \pm 1.7$ y $4.0 \pm 1.7 \mathrm{CR}$ para $T 1, T 2$ y T3, respectivamente). Sin embargo, los promedios recuperados en vaquillas para ET (2.3 $\pm 0.9,1.8 \pm 0.9$ y $1.7 \pm 0.9$ para T1, T2 y T3, respectivamente) y ENT $(1.3 \pm 0.6,0.3 \pm 0.6$ y 0.2 \pm 0.6 para $\mathrm{T} 1, \mathrm{~T} 2$ y $\mathrm{T} 3$, respectivamente) fueron similares a los ET $(1.2 \pm 0.7,2.9 \pm 0.7$ y $1.1 \pm 0.7$ para T1, T2 y T3, respectivamente) y ETN (2.0 \pm $0.5,1.8 \pm 0.5$ y $0.8 \pm 0.5$ para T1, T2 y T3, respectivamente) recuperados en vacas. Así, los promedios para ET y ETN de vacas y vaquillas en el presente estudio indican que vacas y vaquillas tuvieron producciones embrionarias similares debido a una mayor fertilidad de las vaquillas. Coincidiendo con estos resultados otros estudios también han encontrado mayor fertilidad en vaquillas que en vacas ${ }^{(31,36)}$.

No se detectaron diferencias importantes entre tratamientos ( $P>0.05)$ para la variable ET en ninguno de los dos experimentos. Cabe señalar que los promedios de ET para vacas $(1.2 \pm 0.7,2.9 \pm$
0.7 y $1.1 \pm 0.7$ para $\mathrm{T} 1, \mathrm{~T} 2$ y $\mathrm{T} 3$, respectivamente) $\mathrm{y}$ vaquillas $(2.3 \pm 0.9,1.8 \pm 0.9$ y $1.7 \pm 0.9$ para $T 1$, T2 y T3, respectivamente) son similares a los promedios estimados para siete razas de ganado Criollo en Colombia(37). En el estudio mencionado se utilizaron dosis altas y reducidas de $\mathrm{FSH}$, encontrándose rangos de embriones transferibles que fluctuaron entre 0.7 y 2.8 , y entre 0.0 y 3.0 para las dosis alta y baja, respectivamente, sin detectarse diferencias $(P>0.05)$ entre las dosis ${ }^{(37)}$. En general, la información publicada indica que las poblaciones de ganado criollo tienen, en promedio, menor número de embriones transferibles que los que se obtienen en otras razas $B$. taurus y $B$. indicus ${ }^{(9,28)}$. De igual manera, los estudios realizados para evaluar dosis estándar y reducidas de FSH en bovinos criollos, coinciden en señalar que no existen diferencias en la respuesta a la superovulación entre las dosis evaluadas $(37,38,39)$. Es importante enfatizar que el hecho de que no exista diferencia entre tratamientos permite reducir los costos de la superovulación al disminuir la cantidad de FSH sin tener efectos negativos en la respuesta a la superovulación o en la producción embrionaria. En contraste con los resultados del presente estudio, otros autores han encontrado mayores cantidades de embriones transferibles en ganado lechero Criollo tropical de México(39), así como en poblaciones nativas de Irán ${ }^{(14)}$, Corea ${ }^{(15)}$ y Tailandia( ${ }^{(38)}$.

\section{CONCLUSIONES E IMPLICACIONES}

Es factible utilizar dosis reducidas de FSH para la superovulación en vaquillas Criollo Coreño, disminuyendo así los costos de la superovulación y la producción de embriones. Las vacas Criollo Coreño mostraron una mejor respuesta a la superovulación comparadas con las vaquillas; en contraste, las vaquillas tuvieron mayor porcentaje de fertilización. Vacas y vaquillas tuvieron similar producción de embriones.

\section{LITERATURA CITADA}

1. De Alba MJ. El libro de los bovinos Criollos de América. $1^{\mathrm{a}}$ ed. México: Ediciones Papiro Omega SA de CV; 2011.

2. Martínez VG, Montaño BM, Palacios FJA. Efectos genéticos directos, maternos y heterosis individual para tasas de estro, gestación, parición y destete de vacas Criollo, Guzerat y sus cruzas F1. Téc Pecu Méx 2006;44(2):143-154. 
3. Martínez VG, Montaño BM, Palacios FJA. Productividad hasta el destete de vacas Criollo, Guzerat y sus cruzas recíprocas F1. Téc Pecu Mex 2008;46(1):1-12.

4. Martínez VG, Montaño BM. El bovino Criollo del occidente de México. Editores Perezgrovas GRA, de la Torre SJF editores. Los bovinos Criollos de México. Historia, caracterización y perspectivas. México: Universidad Autónoma de Chiapas; 2015:147-174.

5. Asociación de criadores de ganado Criollo Mexicano AC. (ASOCRIOLLO). Memoria técnica de "Curso de acreditación de inspectores de ganado Criollo para registro" Chihuahua, México. 2010.

6. Martínez VG. El ganado bovino Criollo en Nayarit: Ubicación y población estimada. Sitio Experimental El Verdineño. INIFAPCIRPAC. Folleto Técnico N¹. 2005.

7. Hoffmann I, Scherf B. Implementing the Global Plan of Action for Animal Genetic Resources. Recursos Genéticos Animales. FAO 2010;47:1-10.

8. Stroud B, Hasler JF. Dissecting why superovulation and embryo transfer usually work on some farms but not on others. Theriogenology 2006;65-76.

9. Baruselli PS, Sá Filho MF, Martins CM, Nasse LF, Nogueira MFG, Barros CM, Bó GA. Superovulation and embryo transfer in Bos indicus cattle. Theriogenology 2006;(65):77-88.

10. Quezada CA, Avendaño RL, Ramírez GJA, Macías CU, Correa CA. Behavioural, follicular and hormonal characteristics of the oestrus cycle of Mexican Criollo cattle. Anim Prod Sci 2014;54:277-284.

11. Quezada CA, Avendaño RL, Macías CU, Ramírez GJA, Correa CA. Estrus behavior, ovarian dynamics, and progesterone secretion in Criollo cattle during estrous cycles with two and three follicular waves. Trop Anim Health Prod 2014;46:675-684.

12. Lopes de Costa L, Chagas Silva J, Robalo Silva J. Superovulatory response, embryo quality and fertility after treatment with different gonadotrophins in native cattle. Theriogenology 2001;(56):65-77.

13. Quaresma MA, Lopes de Costa $L$, Robalo Silva J. Superovulation of Mertolenga cows with two FSH preparations (FSH-P and FOLLTROPIN). Rev Portuguesa Cienc Vet 2003;(98):81-84.

14. Barati F, Niasari-Naslaji A, Bolourchi M, Sarhaddi F, Razavi K, Naghzali E, Thatcher WW. Superovulatory response of Sistani cattle to three different doses of FSH during winter and summer. Theriogenology 2006;(66):1149-1155.

15. Son DG, Choe CY, Cho SR, Choi SH, Kim HJ, Kim H. The effect of reduced dose and number of treatments of FSH on superovulatory response in CIDR-treated Korean Native cows. J Rep Develop 2007; (53):1299-1303.

16. SAGARPA. Coordinación General de Ganadería. México 2002; http://www.sagarpa.gob.mx/ganaderia/Publicaciones/Paginas/def ault.aspx.

17. Medina GG, Ruiz CJA, Martínez PRA. Los climas de México. Una estratificación ambiental basada en el componente climático. Instituto Nacional de Investigaciones Forestales, Agrícolas y Pecuarias (INIFAP). Libro Técnico № 1. México. 1998.

18. Sistema Estatal de Monitoreo Agroclimático de Nayarit. México 2013. http://www.climanayarit.gob.mx/.

19. Eversole DE, Browne MF, Hall JB, Dietz RE. Body condition scoring beef. Virginia Tech Pub 2009;400-791.

20. Hasler JF. The current status and future of commercial embryo transfer in cattle. Anim Rep Sci 2003;79:245-264.
21. Dulbecco R, Vogt M. Plaque formation and isolation of pure lines with poliomyelitis viruses. J Exp Med 1954;99(2):167-182.

22. International Embryo Transfer Society (IETS). Data retrieval committee report. Embryo Transfer Newsletter 2007;15-20.

23. SAS. SAS User's Guide: Statistics; SAS Inst. Inc., Cary, NC. USA. 2002.

24. Weller JI, Misztal I, Gianola D. Genetic analysis of dystocia and calf mortality in Israeli-Holsteins by threshold and linear models. J Dairy Sci 1988;71:2491-2501.

25. Olesen I, Perez-Enciso M, Gianola D, Thomas DL. A comparison of normal and nonnormal mixed models for number of lambs born in Norwegian sheep. J Anim Sci 1994;72:1166-1173.

26. Matos CAP, Thomas DL, Gianola D, Perez-Enciso M, Young LD. Genetic analysis of discrete reproductive traits in sheep using linear and nonlinear models: II. Goodness of fit and predictive ability. J Anim Sci 1997; 75:88-94.

27. Lentner M, Bishop T. Experimental design and analysis. 2nd ed. USA: Virginia Polytechnic Institute and State University; 1993.

28. Hasler JF. The Holstein cow in embryo transfer today as compared to 20 years ago. Theriogenology 2006;65:4-16.

29. Seidel GE Jr, Elsden PR, Hasler JF. Embryo transfer in dairy cattle. Third ed. USA: Hoards Dairyman; 2003.

30. Matthiesen MM. Effect of donor age on the developmental capacity of bovine cumulus oocyte complexes obtained by repeated OPU from nonstimulated and FSH-superstimulated German Simmental heifers and cows at different life cycle stages [tesis doctoral]. Munich. Alemania: Universidad de Mûnchen; 2011.

31. Donaldson L. Cattle breed as a source of variation in embryo transfer. Theriogenology 1984;21:1013-1018.

32. Breuel KF, Baker RD, Butcher RL, Townsed EC, Inskeep EK, Dailey RA, Lerner SP. Effects of breed, age of donor and dosage of follicle stimulating hormone on the superovulatory response of beef cows. Theriogenology 1991;36:241-255.

33. Yamamoto $T$, Iwata $H$, Goto $H$, Shiratukis $S$, Tanaka $H$, Monji $Y$, Kuwayama T. Effect of maternal age on the developmental competence and progression of nuclear maturation in bovine oocytes. Molecular Rep Develop 2010;77(7):595-604.

34. Su L, Yang S, He X, Li X, Ma J, Wang Y, Presicce GA, Ji W. Effect of donor age on the developmental competence of bovine oocytes retrieved by ovum pick up. Rep Domestic Anim 2012;47(2):184-189.

35. Lerner S, Thayne W, Baker R, Hensche T, Meredith S, Inskeep E, et al. Age, dose $\mathrm{FSH}$ and other factors affecting superovulation in Holstein cows. J Anim Sci 1986;63:176-183.

36. Bodmer M, Janett F, Hassig M, den Daas N, Reichert P, Thun R. Fertility in heifers and cows after low dose insemination with sexsorted and non-sorted sperm under field conditions. Theriogenology 2005;64(7):1647-1655.

37. Estrada JL, Pachón LA, Olivera M, Piedrahita J, Westhusin M. Superovulatory response of Colombian creole cattle to two doses of FSH. Theriogenology 1998;49(1):377-382.

38. Nilchuen $\mathrm{P}$, Chomchai $\mathrm{S}$, Rattanatabtimtong S. Superovulation with different doses of follicle stimulating hormone in Kamphaeng Sean beef cattle. J Anim Vet Adv 2012;11(5):676-680.

39. Rosales MF. Superovulación en ganado lechero Criollo tropical [tesis maestría]. Estado de México, México: Colegio de Postgraduados; 2013. 